Then the conditions on $|x|<1$ require that $f^{1,2}$ should be a solution of (1). If $\operatorname{det}(I-i a) \neq 0$ we can find a solution while if $\operatorname{det}(I-i a)=0$ there exists no solution.

\title{
REFERENCES
}

1. T. Carleman, Sur la résolution de certaines équations intégrales, Mat. Ark. Astronom. Fys. 16, no. 26 (1922).

2. N. I. Muskhelishvili, Singular integral equations, Noordhoff, Groningen, 1953.

3. N. P. Vekua, Systems of singular integral equations and some boundary problems, GITTL, Moscow-Leningrad, 1950.

Carnegie Institute of Technology

\section{A REMARK ON AN ARITHMETIC THEOREM \\ OF CHEVALLEY}

H. BASS

1. Let $k$ be an algebraic number field with ring of integers $\mathcal{O}$, and let $E$ be a finitely generated subgroup of the multiplicative group, $k^{*}$. All but finitely many primes $\mathfrak{p}$ are "prime to $E$," i.e., the units of $\mathcal{O}_{\mathfrak{p}}$ contain $E$. An ideal $\mathfrak{a}$ is called "prime to $E$ " if its prime divisors are. In this case we have a natural homomorphism

$$
E \rightarrow(\mathcal{O} / \mathfrak{a})^{*}
$$

whose kernel, the congruence subgroup $\{a \in E \mid a \equiv 1 \bmod \mathfrak{a}\}$, is evidently of finite index. We denote the group of all (complex) roots of unity by $\boldsymbol{Q} / \boldsymbol{Z}$.

Theorem. Let $\chi: E \rightarrow \boldsymbol{Q} / \boldsymbol{Z}$ be a character of $E$. Then there are infinitely many prime ideals $\mathfrak{p}$ of $k$, prime to $E$, such that $\chi$ factors through a character of $(\Theta / p)^{*}$, i.e., such that $\operatorname{ker}\left(E \rightarrow(\Theta / p)^{*}\right) \subset \operatorname{ker} \chi$.

It follows immediately that if $U$ is a subgroup of finite index in $E$ then $\operatorname{ker}\left(E \rightarrow(\mathcal{O} / \mathfrak{a})^{*}\right) \subset U$ for a suitable $\mathfrak{a}$, which we may take to be square free. This is the form of the theorem proved by Chevalley in [2]. That $\mathfrak{a}$ may be taken square free is implicit in his proof. The following corollary paraphrases Chevalley's theorem.

Corollary 1 (Chevalley). If we embed $E$ in $\prod_{p}$ prime to $E(\theta / p)^{*}$,

Received by the editors August 19, 1964 and, in revised form, September 28, 1964. 
its closure is naturally identical with the completion, $\hat{E}$, of $E$ in the topology defined by all subgroups of finite index.

I was led to these matters after proving the following corollary. I am indebted to J.-P. Serre for referring me to Chevalley's paper.

COROLLARY 2. The algebraic closure of a finite field is generated, as a field, by roots of unity of prime order. The same is (therefore) true of the maximal unramified extension of a p-adic field.

Proof. Let $\overline{\boldsymbol{F}}_{p}$ be the algebraic closure of $\boldsymbol{F}_{p}=\boldsymbol{Z} / p \boldsymbol{Z}$, and let $H$ be the subgroup of $\bar{F}_{p}^{*}$ generated by roots of unity of prime order. Let $L=F_{p}(H)$ and let $G=G\left(\bar{F}_{p} / F_{p}\right)$, the Galois group. To show that $L=\bar{F}_{p}$ it suffices, by Galois theory, to show that the restriction map, $G \rightarrow \operatorname{Aut}(H)$, is a monomorphism, since $L$ is the fixed field of its kernel.

Now $G$ is topologically isomorphic to $\hat{\boldsymbol{Z}}$, with generator $f=$ Frobenius ( $p$ th power). $H$ is isomorphic to the additive group $\oplus_{q \neq p} F_{q}$, so $\operatorname{Aut}(H)=\prod_{q \neq p} F_{q}^{*}$. Under this identification, $G \rightarrow \prod_{q \neq p} F_{q}^{*}$ sends $f$ to the element with all coordinates equal to $p$. With $E$ the subgroup of $Q^{*}$ generated by $p$, our assertion now follows from Corollary 1. Q.E.D.

In case $k=\boldsymbol{Q}$ the theorem above was proved by Mills in [3] in a slightly more precise form. Mills' argument is essentially the same as Chevalley's (of which Mills was presumably unaware). This consists of reducing the theorem to a computation of $\left(F^{*}\right)^{m} \cap k^{*}, F$ being the field over $k$ generated by a primitive $m$ th root of unity. This reduction is repeated, for the reader's convenience, in the next section. The preciseness of the final theorem is then a direct reflection of the precision with which $\left(F^{*}\right)^{m} \cap k^{*}$ is computed.

2. We show here (following Chevalley) how to deduce the Theorem from the next proposition, whose proof will be given in part 3 .

Proposition. Given $N>0$, then there is an $m>0$ such that, if $F$ is the field generated over $k$ by a primitive mth root of unity, we have

$$
\left(F^{*}\right)^{m} \cap k^{*} \subset\left(k^{*}\right)^{N}
$$

Proof of the theorem. Recall that we have $E \subset k^{*}$ and $\chi: E$ $\rightarrow \boldsymbol{Q} / \boldsymbol{Z}$. We must find $\mathfrak{p}$ such that $\operatorname{ker}\left(E \rightarrow(\theta / \mathfrak{p})^{*}\right) \subset \operatorname{ker} \chi$. Choose $N>0$ so that $E \cap\left(k^{*}\right)^{N} C$ ker $\chi$. This is possible since $\chi(E)$ is finite and since $k^{*}$ is the product of a free abelian with a finite group. Now choose $m>0$ as in the proposition above. Then $\left(F^{*}\right)^{m} \cap E \subset \operatorname{ker} \chi$. It follows that $\chi$ factors via $E \rightarrow F^{*} /\left(F^{*}\right)^{m}$; i.e., there is a character $\chi^{\prime}: F^{*} \rightarrow \boldsymbol{Q} / \boldsymbol{Z}$ of order $m$ such that $\chi^{\prime} \mid E=\chi$. Let $L=F\left(E^{1 / m}\right)$, the 
(finite) extension generated by $m$ th roots of elements of $E$. It follows from Kummer theory (see Artin [1]) that there is an $s \in G(L / F)$ such that $s\left(a^{1 / m}\right)=a^{1 / m} \chi(a)$ for all $a \in E$. By the Cebotarev density theorem there exist infinitely many primes $\mathfrak{B}$ of $F$ such that $s=((L / F) / \mathfrak{P})$, the Artin symbol in the abelian extension $L / F$ (see Serre [4, p. 34]). Choose such a $\mathfrak{B}$ prime to $m$. Then if $a \in E$ and $a \equiv 1 \bmod \mathfrak{P}, a$ is an $m$ th power in the local field $F_{\mathfrak{B}}$. Hence the local degrees at $\mathfrak{B}$ of $F\left(a^{1 / m}\right) / F$ are all one. It follows that $s=((L / F) / \mathfrak{B})$ fixes $a^{1 / m}$ and, consequently, $\chi(a)=1$. Thus, the prime $\mathfrak{p}$ of $k$ that $\mathfrak{B}$ divides solves our problem, and we have proved the theorem.

3. The proposition will be proved in a sequence of lemmas which give some more specific information.

Lemмa 1. Let $F / k$ be a finite field extension and $q$ an integer with prime factorization $\prod_{i} p_{i}^{n_{i}}$.

(a) $\left(F^{*}\right)^{q} \cap k^{*}=\bigcap_{i}\left[\left(F^{*}\right)^{p_{i_{i}} n_{i}} \cap k^{*}\right]$.

(b) If $d=[F: k]$ is prime to $q$, then $\left(F^{*}\right)^{q} \cap k^{*}=\left(k^{*}\right)^{q}$.

Proof. (a) is obvious. (b): If $x \in\left(F^{*}\right)^{q} \cap k^{*}$, take norms to obtain $x^{d} \in\left(k^{*}\right)^{q}$. g.c.d. $(d, q)=1 \Rightarrow x \in\left(k^{*}\right)^{q}$.

Now we fix some notation: $k_{m}$ denotes the field over $k$ generated by a primitive $m$ th root of unity.

Lemma 2. If $p \neq 2, \quad\left(k_{p e}^{*}\right)^{p^{n}} \cap k^{*}=\left(k^{*}\right)^{p^{n}} . \quad\left(k_{2 e}^{*}\right)^{2^{n}} \cap k^{*}=\left(k_{2 a}^{*}\right)^{2^{n}} \cap k^{*}$ $\subset\left(k^{*}\right)^{2^{n-1}}$, where $a=\min (2, e)$. Hence, if $k_{4} \subset k,\left(k_{2^{e}}\right)^{2^{n}} \cap k^{*}=\left(k^{*}\right)^{2^{n}}$.

Proof. See Chevalley [2, pp. 37, 38].

For a prime $p$ we define

$$
e(p)=e(p, k)
$$

to be the largest integer $e$ such that, for each prime $p$ of $k$ above $p$, the local field at $p$ contains $k_{p}$. Note that if $e>0$ and $p \neq 2$ this implies $p$ is ramified; hence $e(p)=0$ for all but finitely many $p$.

Lemma 3. Suppose $n \geqq e=e(p)$. Then for any $m>0$

$$
\left(k_{m}^{*}\right)^{p^{n}} \cap k^{*} \subset\left(k_{p e}^{*}\right)^{p^{n-\bullet}} \cap k^{*},
$$

unless $p=2$ and $e=1$. In this case replace the right side by $\left(k^{*}\right)^{2^{n-2}}$.

Proof. Write $m=p^{r} q$ with $q$ prime to $p$. We apply Lemma 2 to $k_{q}$ to obtain $\left(k_{m}^{*}\right)^{p^{n}} \cap k_{q}^{*} \subset\left(k_{q}^{*}\right)^{p^{h}}$, where $h=n$ for $p$ odd, and which we discuss below for $p=2$. Choose $f$ maximal so that $k_{p j} \subset k_{q}$. If $F$ is the local field of $k$ at a prime dividing $p$ then $F \subset F_{p} \subset F_{q}$. However, the big extension is unramified, and the small one totally rami- 
fied. Hence $F=F_{p^{f}}$ so, by definition of $e=e(p)$, we have $f \leqq e$.

Suppose $y \in k_{q}^{*}$ is such that $y^{p^{h}} \in k_{p^{f}}$. If $s \in G\left(k_{q} / k_{p^{s}}\right)$ then $s y=y z$ with $z^{p^{h}}=1$. By definition of $f$, therefore, $z^{p^{f}}=1$. It follows that $s y^{p^{j}}=y^{p}$, so $y^{p^{f}} \in k_{p s}$. Writing $y^{p^{h}}=\left(y^{p^{f}}\right)^{p^{h-f}}$ we have therefore shown that

$$
\left(k_{q}^{*}\right)^{p^{h}} \cap k_{p^{f}}^{*} \subset\left(k_{p^{f}}^{*}\right)^{p^{h-f}} \subset\left(k_{p e}^{*}\right)^{p h-\epsilon},
$$

the second inclusion ensuing from $f \leqq e$. We have thus descended the field tower, $k \subset k_{p^{f}} \subset k_{q} \subset k_{m}$, and proved our assertion in the case $h=n$. By Lemma 2 this is the case for $p$ odd, and for $p=2$ provided $k_{4} \subset k_{q}$. In the remaining case we must have $p=2$ and $f=1$, so $k_{p f}=k$ and we can take $h=n-1$. The proof then yields $\left(k_{m}^{*}\right)^{2^{n}} \cap k^{*} \subset\left(k^{*}\right)^{2^{h-1}}$ $=\left(k^{*}\right)^{2^{n-2}}$.

Combining Lemmas 1,2 , and 3 we have:

Corollary. Let

$$
f(p)= \begin{cases}e(p) & \text { for } p \neq 2, \\ e(2)+2 & \text { for } p=2 .\end{cases}
$$

Then if $m$ has prime factorization $\prod_{p \in S} p^{n(p)}$, with $n(p) \geqq f(p)$, and if $m_{0}=\prod_{p \in S} p^{f(p)}$, then

$$
\left(k_{m}^{*}\right)^{m} \cap k^{*} \subset\left(k^{*}\right)^{m / m_{0}} .
$$

Since $m_{0}$ depends only on the prime divisors of $m$, and not their exponents, it is clear that the proposition of $\$ 2$ follows from the corollary.

\section{REFERENCES}

1. E. Artin, Galois theory, Univ. Notre Dame, Notre Dame, Ind., 1944.

2. C. Chevalley, Deux théorèmes d'arithmétique, J. Math. Soc. Japan 3 (1951), $36-44$.

3. W. H. Mills, Characters with preassigned values, Canad. J. Math. 15 (1963), 169-171.

4. J.-P. Serre, Corps locaux, Hermann, Paris 1962.

Columbia University 